\title{
Coarse Symbol Timing Synchronization Improved Algorithm for CMMB Mobile TV
}

\author{
Lei Guoping ${ }^{1, *}$, Xie Hong ${ }^{2}$ and Tan Zefu ${ }^{1}$ \\ ${ }^{I}$ Key Laboratory of Signal and Information Processing, Chongqing Three Gorges University, Wanzhou, Chongqing, \\ 404100, China \\ ${ }^{2}$ Department of Mechanical and Electronic Engineering, Chongqing Three Gorges Vocational College, Chongqing, \\ 404155, China
}

\begin{abstract}
There are some multipath and frequency offset in wireless transmission environment, in order to determine the correct starting position of each OFDM symbol, CMMB system must achieve accurate symbol synchronization for sending and receiving data, making FFT data transform window position in receiver consistent with IFFT window position in sender. This paper combined the specific frame structure of CMMB standard protocol, analyzed some influences of symbol synchronization timing errors in system, discussed the traditional ML (Maximum Likelihood) algorithm, the non data aided algorithm and the reverse conjugate symmetry direct algorithm, and based on the characteristics of these algorithm, proposed a coarse symbol timing synchronization improved algorithm according to CMMB mobile TV, the feasibility of this algorithm is verified in AWGN and multipath channel. Simulation results show that the performance of improved algorithm is better, there is no multi-peak of ML algorithm, and don't worry about appearing "lat roof" phenomenon like no data aided algorithm, and it also eliminates adjacent peak interference of the reverse conjugate symmetry direct algorithm, this algorithm is not limited by SNR, even in bad multipath channel condition, the approximate starting position of symbol can be estimated.
\end{abstract}

Keywords: Reverse conjugate symmetry, Coarse symbol timing synchronization, OFDM, CMMB.

\section{INTRODUCTION}

Digital is a new technology revolution all over the world, its rapid development and change make the development fast in radio and TV industry. Radio and TV should be the fastest, most convenient and most popular of culture entertainment information tool at present in our country, the China Mobile Multimedia Broadcasting (CMMB) is a new technology application in new field. it is brought by digital TV, filled the blank of mobile service, with the characteristics of popularization, fast, low cost, especially the dissemination of traditional way, CMMB has congenital advantage in the general services for public [1].

CMMB system introduces OFDM modulation technology, although OFDM symbol has cyclic prefix, the symbol synchronization requirements are reduced, but synchronous timing point does not necessarily fall in cyclic prefix within no inter-symbol interference in complicated multipath environment, resulting in ISI, influencing the performance of the whole communication system. So realizing the symbol synchronization is the key to correct demodulation data in CMMB system, and it is the recondition of channel estimation and carrier frequency offset estimation, needs to be done first. In order to realize symbol synchronization more accurate, This paper analyzed the traditional ML algorithm, the non data aided algorithm and the reverse conjugate symmetry direct algorithm, then proposed the coarse symbol synchronization improved algorithm, which is based on frame structure of CMMB system sync signal. Compared with traditional algorithm, the correlation peak of improved algorithm is sharper, there is no multi-peak of ML algorithm, and don't worry about appearing "lat roof" phenomenon like no data aided algorithm, and it also eliminates the adjacent peak interference of the reverse conjugate symmetry direct algorithm, and it also has good performance in bad multipath channel conditions.

\section{THE FRAME STRUCTURE OF CMMB SYSTEM}

CMMB system uses own standard technology, so its frame structure is different from other multimedia standard, as shown in Fig. (1). The length of a signal frame at physical layer is 1 second, and each frame is divided into 40 slots, including a beacon and 53 OFDM symbols, each slot is $25 \mathrm{~ms}$, its beacon is composed by an ID signal from transmitter (TxID) and two identical sync signals [2], these sync signals are long PN sequence known, It can realize synchronization effectively and quickly, this is an important characteristic of frame structure, the most prominent place is also different from other broadcast frame [3-5]. 


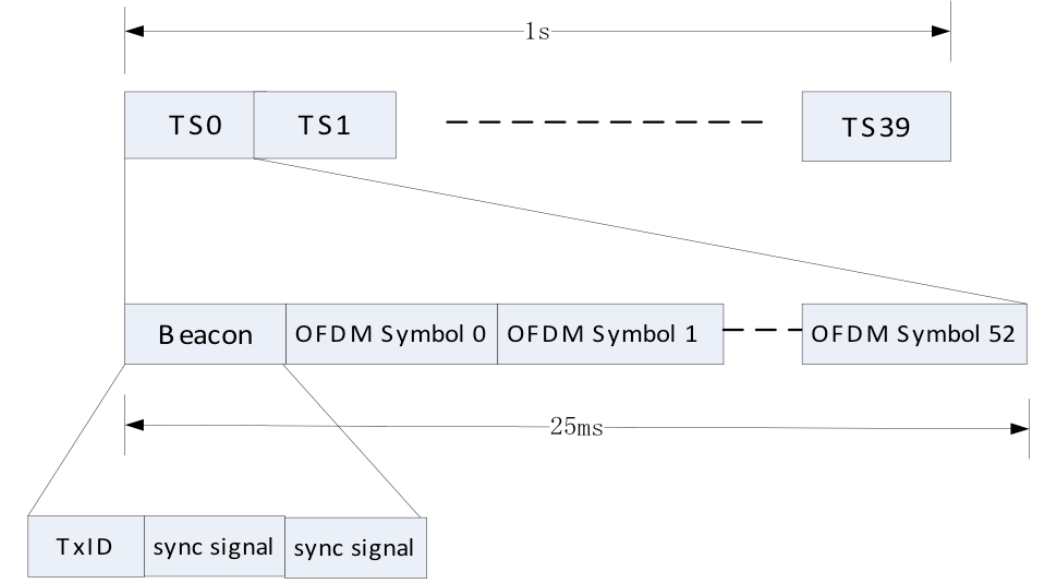

Fig. (1). Frame structure of CMMB system.

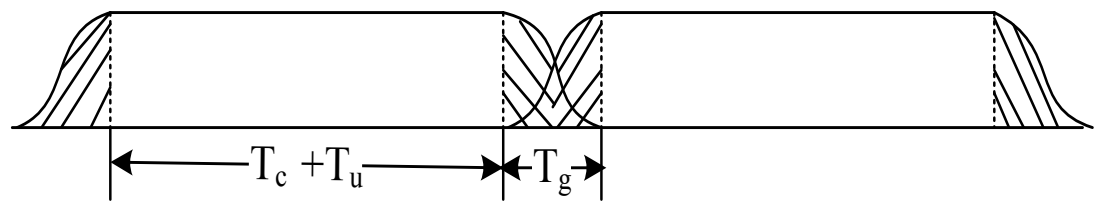

Fig. (2). Symbols' GI overlapping.

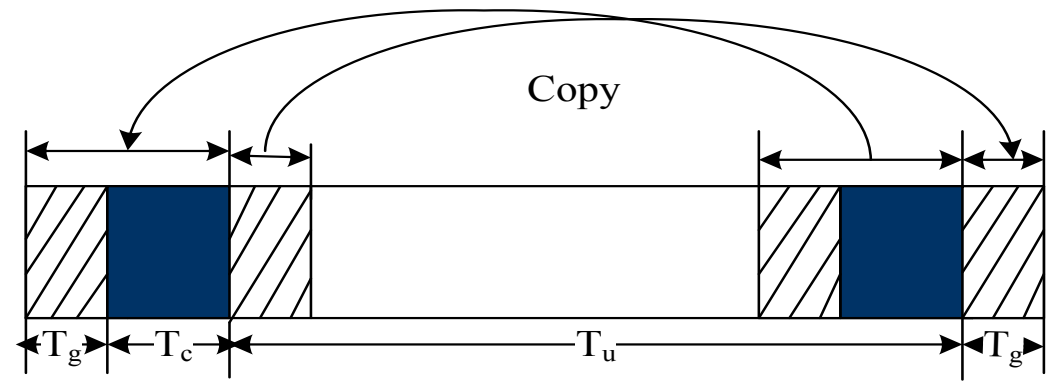

Fig. (3). Structure of CP and GI.

The data OFDM symbols in each slot are inserted a cyclic prefix $(\mathrm{CP})$ as guard interval for CMMB system, while there are some guard intervals (GI) between transmitter's ID signal and sync signals, they also exist between two OFDM symbols, setting window function to make an overlap for the GI of a former symbol's tail and the GI of a latter symbol's head, that is $T_{g}$ in Fig. (2). When overlaping, two sync signals in each slot are processed as whole, so there is no GI between two sync signals.

The structure of CP and GI signals is shown in Fig. (3), where $T_{c}$ is the length of $\mathrm{CP}, T_{g}$ is the length of GI, and $T_{u}$ is data volume, GI and CP are replication of the end of useful data symbols.

\section{THE COARSE SYMBOL SYNCHRONIZATION ALGORITHM}

The CMMB system is seen as one of traditional OFDM broadcast system, so some symbol sync method for OFDM system can also be used for CMMB system, but the estimation performance of different methods are different. The realization of synchronization is the precondition of channel estimation and frequency offset estimation, so the coarse synchronization should be done first in synchronous system.

\subsection{The Traditional ML Algorithm}

The traditional ML algorithm [6] utilizes redundant information carried by $\mathrm{CP}$ to make correlation operation to determine correct starting position of symbols. Assuming transmitted signal $s(n)$ goes through Gaussian channel in CMMB system, the received signal is:

$r(n)=s(n-\theta) e^{j\left(\frac{2 \pi \Delta n_{n}}{N}+\varphi_{0}\right)}+n(k)$

Where $\boldsymbol{\theta}$ is symbol offset, $\Delta f=\left(f_{c}^{\prime}-f_{c}\right) \times T_{u}$ is frequency offset which is normalized, $n(k)$ is additive white gauss noise.

The main calculation steps of ML algorithm is: 
$\varphi(\theta)=\frac{1}{2} \sum_{k=\theta}^{\theta+L-1}|r(k)|^{2}+|r(k+N)|^{2}$

$\gamma(\theta)=\sum_{k=\theta}^{\theta+L-1} r(k) r^{*}(k+N)$

$\rho=\frac{\sigma_{s}^{2}}{\sigma_{s}^{2}+\sigma_{n}^{2}}=\frac{S N R}{S N R+1}$

$\Lambda(\theta, \Delta f)=|\gamma(\theta)| \cos (2 \pi \Delta f+\arg [\gamma(\theta)])-\rho \varphi(\theta)$

Where $\mathrm{N}$ is the length of effective OFDM symbol, $\mathrm{L}$ is the length of CP, In order to make calculation more simple, equation (5) can be simplified as:

$\hat{\theta}_{M L}=\underset{\theta}{\arg \max }\{|\gamma(\theta)|-\rho \varphi(\theta)\}$

By (4) (6) shows, when ML algorithm achieves coarse symbol synchronization estimation, We must get SNR information, so it is often difficult to achieve by only using time domain algorithm of symbol synchronization. While ML algorithm executes each operation, need calculate square amplitude two times, and each square amplitude computing is equivalent to real multiplication two times. This algorithm also need calculate a complex multiplication, and each complex multiplication is equivalent to real multiplication four times. Finally it will also carry out amplitude operation one time. So ML algorithm equivalent calculation is: solving complex amplitude is one time, real multiplication is nine times. Obviously, this algorithm's computation is larger [7]. The MC (Maximum Correlation) detection algorithm is improved [8], its computation complexity is decreased, but the symbol start position is estimated by this algorithm has greater volatility, the estimation performance also declined.

\subsection{The Non Data Aided Algorithm}

By the preceding CMMB system frame structure analysis, we know that each slot has two identical sync signal in frame structure, and these sync signals $S_{b}(n)$ are given by formula (7), they are pseudo random signals, and frequency band is limited, there $N_{b}=2048$ is the number of sync signal sub-carrier ( $8 \mathrm{MHz}$ model), $X_{b}(k)$ is BPSK modulation signals bearing binary pseudo random sequence in frequency domain.

$S_{b}(n)=\frac{1}{N_{b}} \sum_{k=0}^{N_{b}-1} X_{b}(k) e^{j 2 \pi k n / N_{b}}$

In time domain, when there is normalized carrier frequency offset $\Delta F$, and initial phase deviation $\Delta \Phi$, it has relation between the received sync signal $y(n)$ and the sent sync signal $S_{b}(n)$ (do not consider noise), it is as follows:

$y(\mathrm{n})=S_{b}(\mathrm{n}) e^{j 2 \pi \Delta F n / N_{b}+j \Delta \Phi} \quad n=0,1 \cdots N_{b}-1$
Because of the sync signal is pseudo random sequence, and its correlation is very strong, for the CMMB system, the literature [9] proposed to use two sync signal in slot structure, using the non data aided algorithm to conduct correlation operation on the received signal, its estimation algorithm is:

$D(n)=\sum_{n=k}^{k+N_{b}-1} y^{*}(n) y\left(n+N_{b}\right) \quad k=1,2, \mathrm{~L}, \infty$

When $D(n)$ has a peak, the corresponding sampling point $n$ is the starting position of slot sync signal, by formula (9) shows, the computation quantity of this algorithm is relatively small, but the correlation peak is not obvious, especially in multipath environment, the correlation peak has "hillside", a performance simulation figure can be seen latter.

\subsection{The Reverse Conjugate Symmetry Direct Algorithm}

The literature [10] proposed algorithm is based on non data aided algorithm, derived a coarse symbol synchronization algorithm which is using two identical sync signals in time domain, it is according to the beacon structure in CMMB standard (It is showed in Fig. 1). Obviously, the sync signal $S_{b}(n)$ in formula (7) is IFFT of $X_{b}(k)$, because $X_{b}(k)$ is a real sequence, it is based on the basic knowledge of Digital Signal Processing (DSP) [11], we can get:

$$
\begin{aligned}
& S_{b}^{*}(n)=\left(\frac{1}{N_{b}} \sum_{k=0}^{N_{b}-1} X_{b}(k) e^{j 2 \pi k n / N_{b}}\right)^{*}=\frac{1}{N_{b}} \sum_{k=0}^{N_{b}-1} X_{b}^{*}(k) e^{-j 2 \pi k n / N_{b}} \\
& =\frac{1}{N_{b}} \sum_{k=0}^{N_{b}-1} X_{b}(k) e^{j 2 \pi k(-n) / N_{b}}=S_{b}(-n) \\
& S_{b}\left(N_{b}-n\right)=\frac{1}{N_{b}} \sum_{k=0}^{N_{b}-1} X_{b}(k) e^{j 2 \pi k\left(N_{b}-n\right) / N_{b}} \\
& =\frac{1}{N_{b}} \sum_{k=0}^{N_{b}-1} X_{b}(k) e^{j 2 \pi k(-n) / N_{b}}=S_{b}(-n)
\end{aligned}
$$

Comparing (10) and (11), we know that $S_{b}^{*}(n)=S_{b}\left(N_{b}-n\right) \quad$, where $n=0,1, \cdots, N_{b}-1 \quad$, and $n \neq N_{b} / 2, n \neq 0$, so when a frequency sync signal in CMMB system slot finishes IFFT, the time domain signal obtained has reverse conjugate symmetrical characteristic, the data characteristic of two sync signal in beacon can be represented by equation (12):

$\left[\begin{array}{llll}A & A_{\text {reverse }}^{*} & A & A_{\text {reverse }}^{*}\end{array}\right]$

In $8 \mathrm{MHZ}$ mode, the main function of the reverse conjugate symmetry direct algorithm is:

$$
\begin{aligned}
& R(d)=\sum_{k=0}^{N_{b} / 2-1} r^{*}(d+k) r(d-k-1) \\
& C(d)=\sum_{k=0}^{N_{b} / 2-1}|r(d+k)|^{2}
\end{aligned}
$$




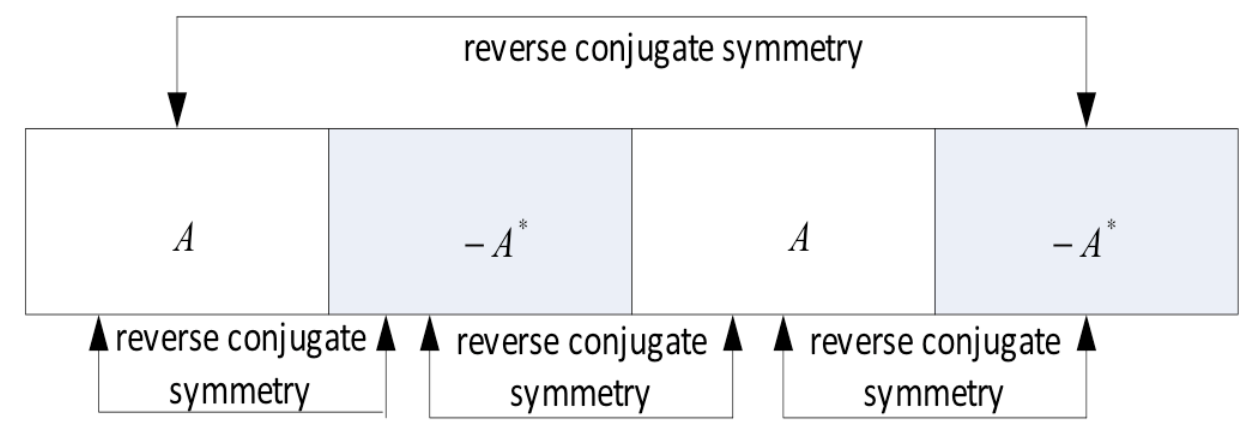

Fig. (4). Decomposition of a synchronization block

$$
P(d)=\frac{|R(d)|^{2}}{C(d)^{2}}
$$

\subsection{The Reverse Conjugate Symmetry Improved Algo- rithm}

In order to get correlation peak more obvious, synchronous timing point is more accurate, this paper proposed the reverse conjugate symmetry improved algorithm, which is based on the reverse conjugate symmetry direct algorithm, and combined the specific frame structure of CMMB standard protocol.

$A_{\text {reverse }}^{*}$ is the reverse conjugate symmetry of $A$ from the equation (12), then the synchronization block in beacon can be decomposed into four equal length data block, as shown in Fig. (4), In $8 \mathrm{MHZ}$ mode, the length of each data block is $N_{b} / 2=1024$.

For CMMB system, if $N_{b} / 2$ data points in neighboring position conduct reverse conjugate symmetry operation, the sampling point is corresponding to the maximum correlation value is regarded as a sync timing point, there are several sync timing point appearing, and there is a transmitter identifier in front of the sync signals, it also has a reverse conjugate symmetry, the interference may be produced possibly, it can make an error for synchronous timing, as shown in Fig. (5), there are three large peak, it will increase the system complexity. So, in order to improve the reliability of timing estimation, reduce its complexity, this paper chooses the first and the last block in Fig. (4) to conduct reverse conjugate symmetry operation.

In $8 \mathrm{MHz}$ model, it is assumed that the mobile position of the current slide relation is $d$, the coarse symbol synchronization improved algorithm is based on sync signal in slot structure, the main timing estimation function is:

$$
\begin{aligned}
& R(d)=\sum_{n=1}^{N_{b} / 2-1} y(d+n) y(d+4097-n) \\
& C(d)=\sum_{n=1}^{N_{b} / 2-1}|y(d+n)|^{2}
\end{aligned}
$$

$$
P(d)=\frac{|R(d)|^{2}}{C(d)^{2}}
$$

When the function $P(d)$ has a maximum slope peak, it can obtain the coarse symbol timing estimation value $d$.

\section{PERFORMANCE SIMULATION AND ANALYSIS}

CMMB standard gives two kinds of physical bandwidth, $8 \mathrm{MHz}$ and $2 \mathrm{MHz}$. In this paper, all simulations are based on $8 \mathrm{MHz}$. At this time, the head of first sync signal is away from the head of slot in each slot, it's 408 sub-carriers, in order to verify the feasibility of this algorithm,we simulated all synchronization algorithm in AWGN channel or in multipath channel, the simulation test conditions are such as Table 1.

Supposing system sampling clock synchronization precision, but it exists carrier frequency offset (frequency offset is normalized), it is $\Delta F=2.45$, the maximum doppler frequency shift is $f_{d}=30 d p$, the signal noise ratio is $S N R=10 d B$, the time delay is $4.5 \mu \mathrm{s}$, the performance simulation of $\mathrm{ML}$ symbol timing estimation algorithm in AWGN channel is shown as Fig. (6).

Table 1. The simulation conditions.

\begin{tabular}{|c|c|}
\hline The Time Slots Number & $\mathbf{4 0}$ \\
\hline \hline The system physical bandwidth & $8 \mathrm{MHz}$ \\
\hline The symbol sub-carrier number & 4096 \\
\hline The effective number of sub-carriers & 3076 \\
\hline OFDM cyclic prefix & $512(1 / 8)$ \\
\hline Continuous pilot & 82 \\
\hline Discrete pilot & 384 \\
\hline Constellation mapping & QPSK \\
\hline Guard interval & $2.4 \mu \mathrm{s}$ \\
\hline
\end{tabular}




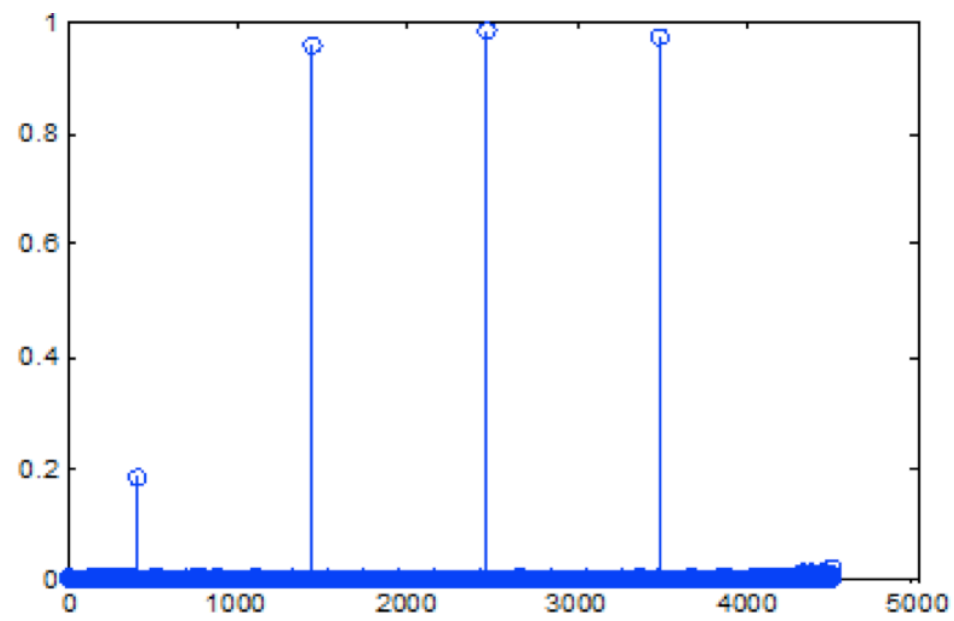

Fig. (5). The multiple correlation peak of neighboring data block.

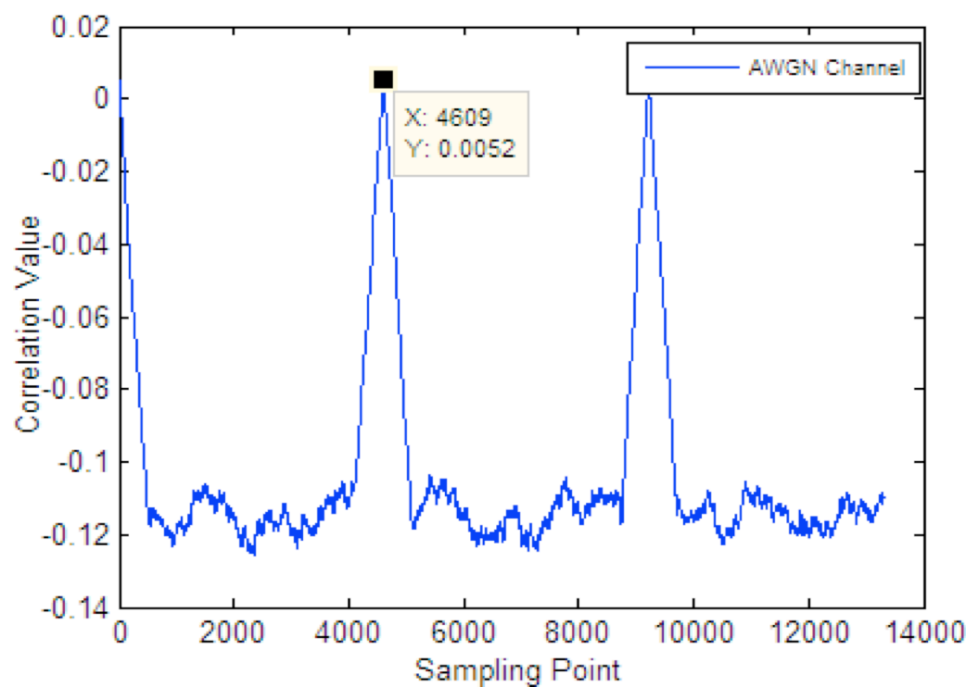

Fig. (6). ML symbol timing estimation algorithm.

Generally, the position of maximum correlation peak in simulation algorithm is estimated as the position of symbols. The digital displays in Fig. (6) is a sampling point of the second OFDM symbol, which is in the case of removing sampling points of beacon structure in slot, it is consistent with the expected value. Predictably, when frequency offset is small in AWGN channel, the ML algorithm can easily find the starting position of symbols, but when OFDM symbols conduct sliding correlation, a peak value near the correlation peak does not have significant difference, and it has no discrete single-peak. Especially when frequency offset is high, and the multipath channel condition is poor, the sharpness of peak will be even worse, and it may appear "flat", so we will not be able to accurately find the starting position of peak at this time.

Under the same conditions, the simulation of the non data aided algorithm is shown in Fig. (7), value $d=414$ is the starting point for the first sync signal in slot structure, comparing with the theoretical value $d=408$, it has only 6 sampling points difference. So, in AWGN channel, this algorithm can find the approximate position, but the correlation peak is "flat", when detecting the maximum peak, it is easy to get error, the flatness of slope peak may be stronger in multipath channel, leading to generate correlation between the received symbol data with the neighboring position data, causing ISI, which is bad for determining the starting position of symbol.

In AWGN channel, a simulation of the reverse conjugate symmetry direct algorithm is shown in Fig. (8), which is put forward in literature [10], this algorithm can find the final approximate position of sampling points for the first sync signal, it is $m=2461$, comparing with the theoretical value $m=2456$, it has 5 sampling points difference, the different value is allowed in CMMB system. The figure shows that the correlation peak of simulation algorithm is relatively sharp, in bad multipath channel, it also can estimate the approximate starting position of symbols, as shown in Fig. (9), 


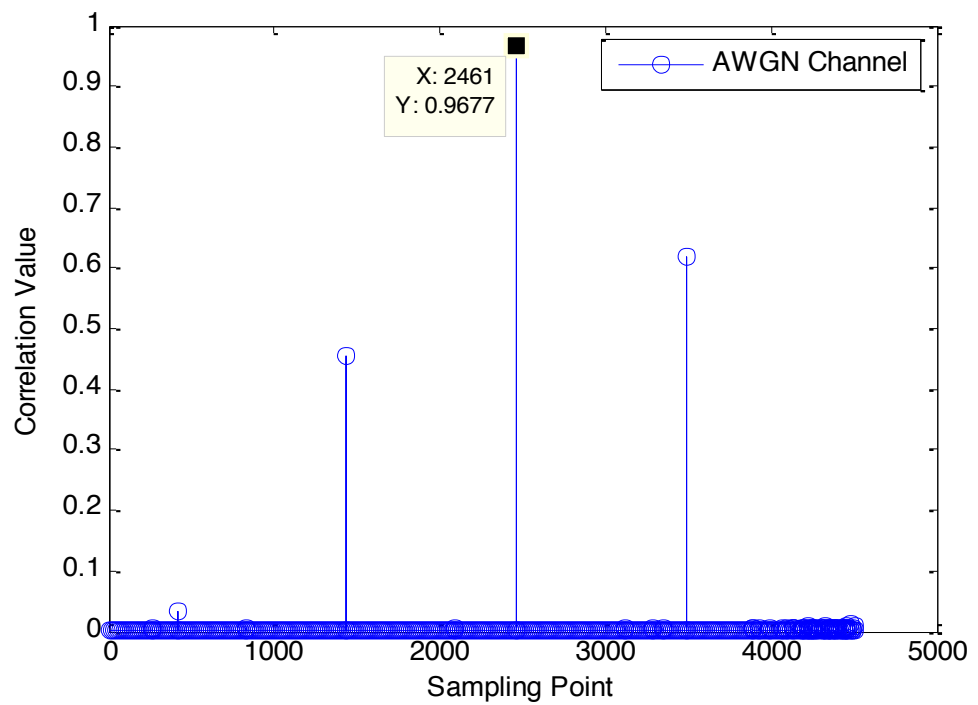

Fig. (7). The non data aided algorithm in AWGN channel.

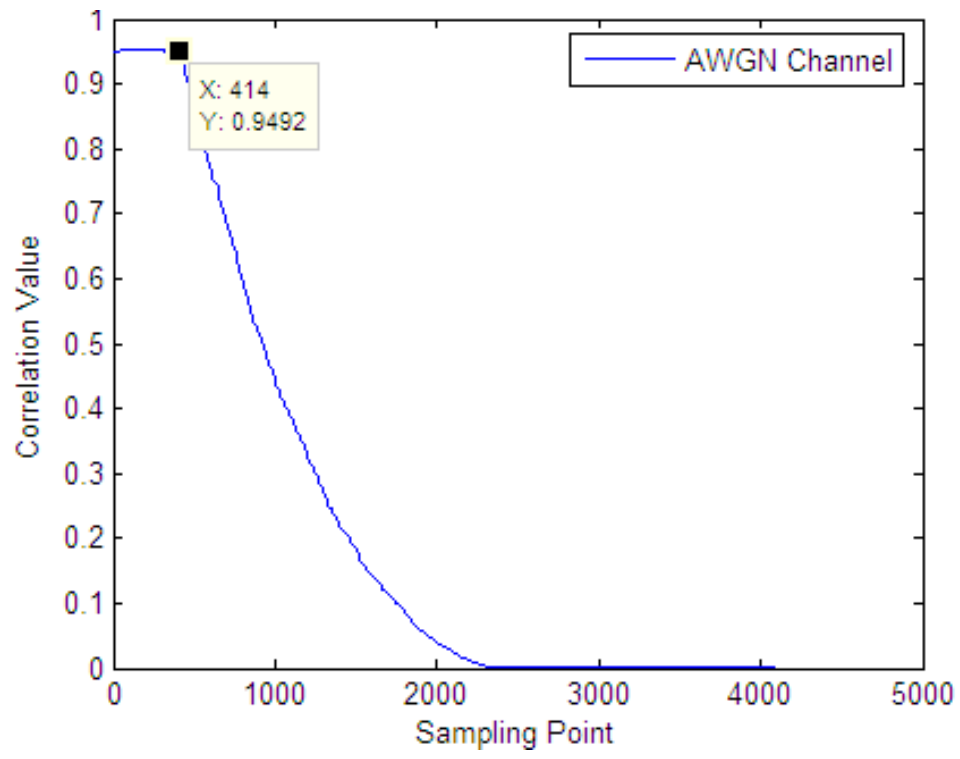

Fig. (8). The reverse conjugate symmetry direct algorithm in AWGN channel.

which multipath channel is the first multipath of equation (19). We can see the correlation peak is sharp, only appearing small fluctuation in some sampling points. But it is obvious that, this algorithm simulation appears disturbance near the maximum correlation peak both in AWGN channel and multipath channel, these slope peaks are also sharp, if it is in very bad condition, The sync signal may be misjudge, that can affect the accuracy of the receiving for CMMB data.

This paper proposed a reverse conjugate symmetry improved algorithm, the simulation is showed in Fig. (10), obviously, the correlation peak is very sharp, which has discrete single peak characteristic, and it can find the starting position of the first sync signal $d=408$, it is consistent with the theoretical value, the estimation performance is very good. So we don't worry about "flat" like non data aided algorithm, it also eliminates the adjacent peak interference of the reverse conjugate symmetry direct algorithm, and this algorithm is not limited by SNR, even in bad multipath channel, it also can estimate the approximate starting position of symbol, as shown in Fig. (11). Each channel is composed of six paths, the channel parameters are as follows:
H1 $\left[\begin{array}{cccccc}0 & 0.9 & 3.0 & 25.9 & 26.4 & - \\ 0 & -17.6 & -24.4 & -6.6 & -16.6 & -\end{array}\right]$
H3 $\left[\begin{array}{cccccc}0 & 3.0 & 4.3 & 19.9 & 22.9 & 24.1 \\ -0.5 & -0.3 & -5.5 & 0 & -4.2 & -5.4\end{array}\right]$
B2 $\left[\begin{array}{cccccc}0 & 0.9 & 2.5 & 4.2 & 5.9 & 9.5 \\ -0.1 & 0 & -8.3 & -18.5 & -18.5 & -13.1\end{array}\right]$
H2 $\left[\begin{array}{ccccccc}0 & 3.6 & 5.3 & 19.2 & 22.8 & 24.0 \\ -7.9 & 0 & -2.7 & -10.2 & -0.7 & -7.0\end{array}\right]$
B1 $\left[\begin{array}{llllll}0 & 0.5 & 1.3 & 2.2 & 3.0 & - \\ -10.2 & 0 & -8.9 & -17.5 & -22.0 & -\end{array}\right]$
B3 $\left[\begin{array}{cccccc}0 & 0.8 & 2.2 & 5.0 & 7.7 & - \\ -3.9 & 0 & -1.9 & -14.7 & -7.6 & -\end{array}\right]$ 


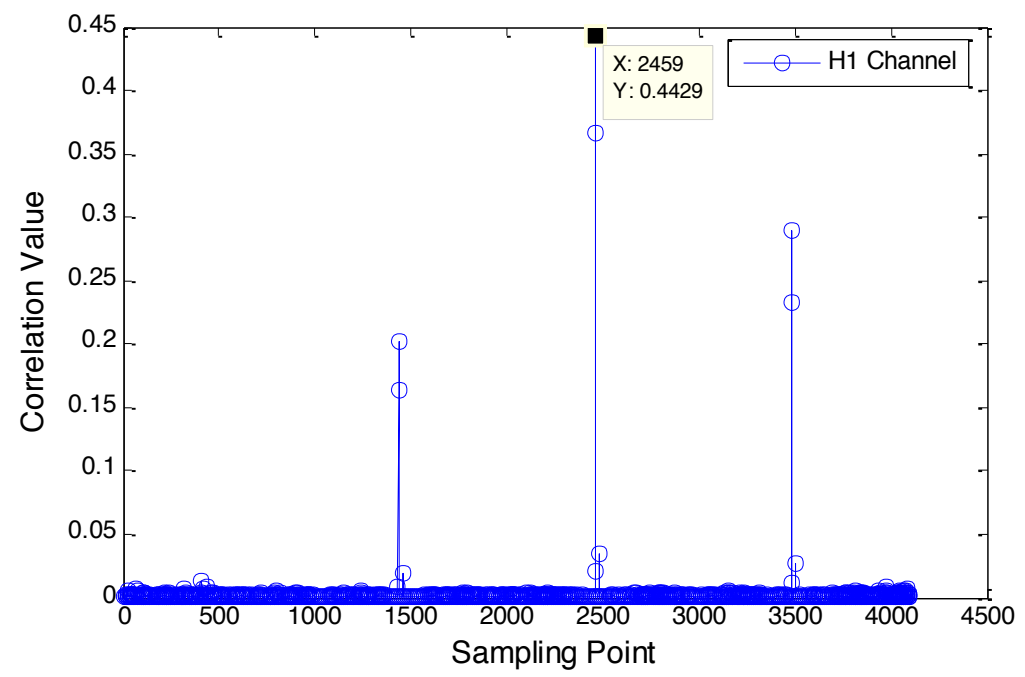

Fig. (9). The reverse conjugate symmetry direct algorithm.

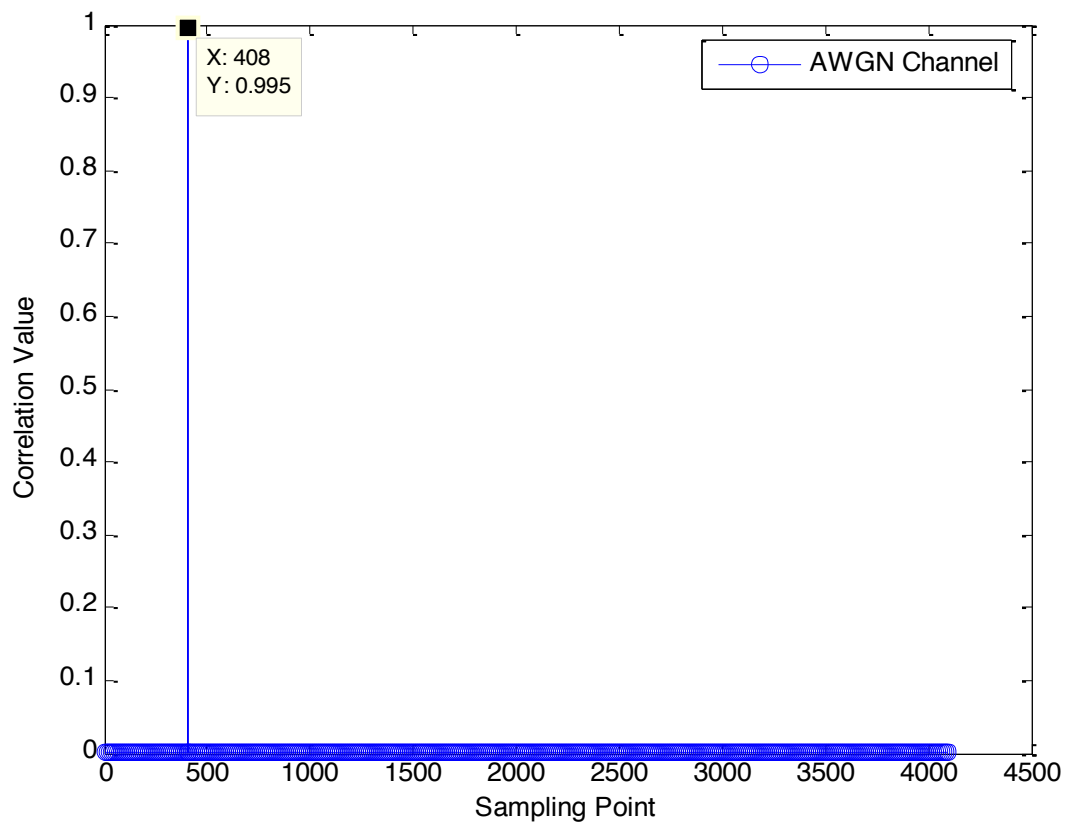

Fig. (10). The reverse conjugate symmetry in multipath channel improved algorithm in AWGN channel.

The first line of each group in equation (19) is the delay time, the unit is $\mu s$, the second line indicates the average power in main path, the unit is $d B$, the range of SNR is $0 \sim 20 d B$, it can be seen from the Fig. (11), even in multipath fading channel, the correlation peak of the improved algorithm is also very sharp, only a small ups and downs appears in some sampling points, the estimation performance will not be influenced. Therefore, this algorithm is particularly suitable for burst transmission mode, when a new slot arrives, this algorithm can quickly capture the long synchronization sequences, and generate larger relative gain, then the position of OFDM symbol is estimated.

\section{CONCLUSION}

This paper is according to the characteristic of CMMB system, it is based on the analysis of the traditional ML, the non data aided algorithm, and the reverse conjugate symmetry direct algorithm, then proposing a new coarse symbol synchronization improved algorithm, which makes use of the reverse conjugate symmetry property of synchronization sequence in frame structure. In AWGN channel, when SNR is low, the simulation result shows that the timing estimation algorithm is proposed is superior to the non data aided algorithm. and there is no multi-peak interference like direct algorithm. It also can obtain a good performance in multipath channel, fully meet requirements of CMMB system, espe- 

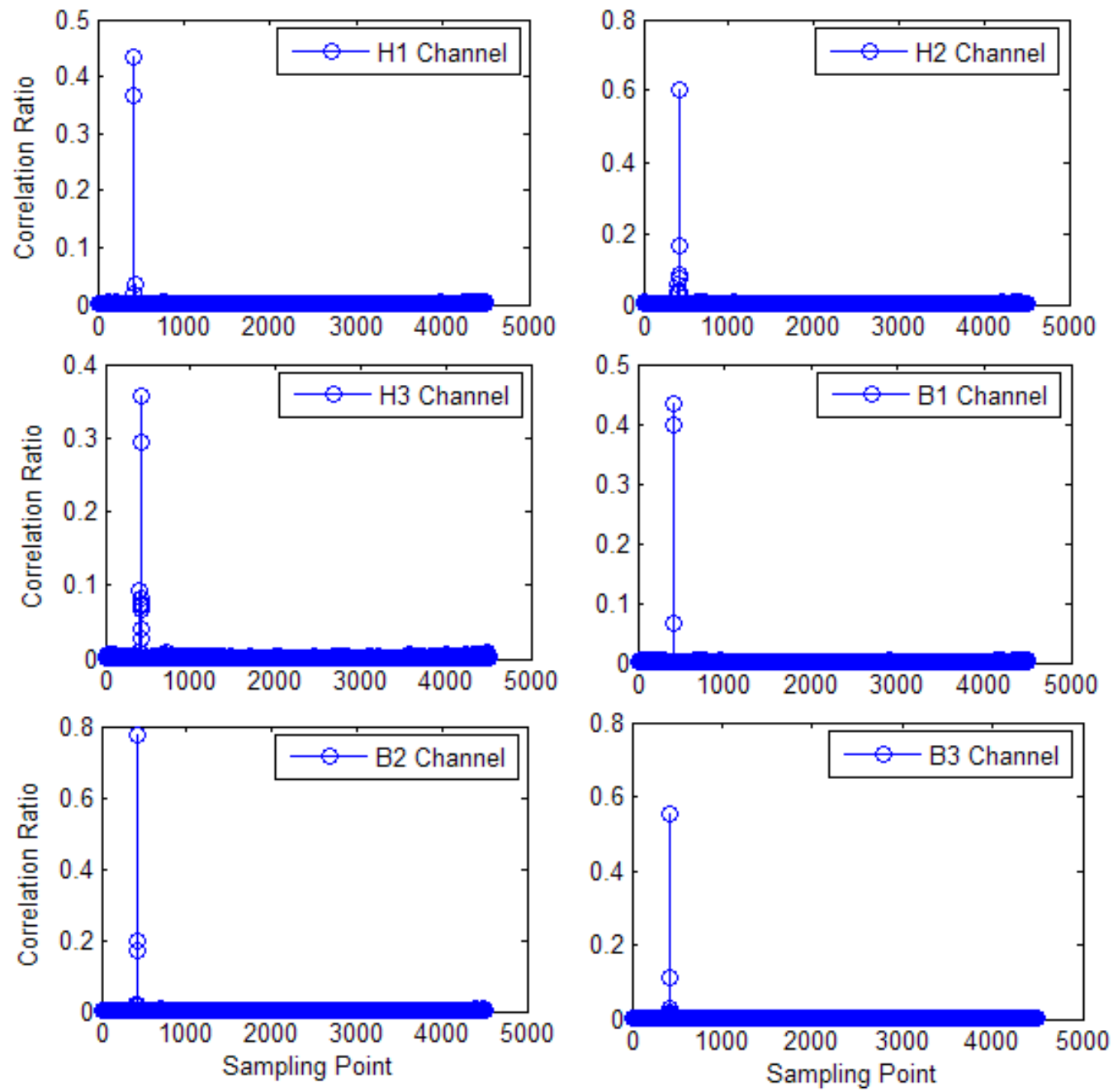

Fig. (11). The reverse conjugate symmetry improved algorithm in multipath channel.

cially it is suitable for OFDM coarse symbol synchronization in CMMB system. In the further, we can further refine this work, design different solutions for system's synchronization, and make further exploration deeply.

\section{CONFLICT OF INTEREST}

The author confirms that this article content has no conflict of interest.

\section{ACKNOWLEDGEMENTS}

This work was supported by the project Program for Innovation Team Building at Institutions of Higher Education in Chongqing (Grant No.KJTD201320); Project Supported by Achievement Transfer Program of Institutions of Higher Education in Chongqing (Grant No.KJZH14214); Project Supported by Scientific and Technological Research Program of Chongqing Municipal Education Commission (Grant KJ131118); Project Supported by Open fund of Chongqing Three Gorges University of Key Laboratory of Signal and Information Processing.

\section{REFERENCES}

[1] J. Song, Z. Yang and L. Yang, "Technical review on chinese digital terrestrial television broadcasting standard and measurements on some working modes", IEEE Transactions on Broadcasting, vol. 53, no.1, pp.1-7, 2007.

[2] W. Zheng. "The standard GY/T 220.1-2006 of Radio, Film and TV industry in China", The Broadcasting Channel Frame Structure, Channel Coding and Modulation in CMMB", 2006.

[3] Y. Wang, "The Research on Synchronization Technique in Wireless OFDM Transmission System", Xi'an Electronic and Science University, 2006.

[4] M. Speth, S.A. Fechtel and G. Foek, "Optimum receiver design for wireless broad-band system using OFDM-Part I", IEEE Transaction on Communications, vol. 47, no. 11, pp. 1668-1677, 1999.

[5] Z. Liu, "The Research on Frame Synchronization and Symbol Synchronization Technology for CMMB System", Shanghai Jiao Tong University, 2008.

[6] T. Lv and J. Chen, "ML estimation of timing and frequency offset using multiple OFDM symbols in OFDM systems", IEEE Global Telecommunications Conference, GLOBECOM' '03, 2003, pp. 2280 - 2284

[7] T. Keller, L. Piazzo, P. Mandarini, and Hanzo, "Orthogonal frequency division multiplex synchronization techniques for frequency selective fading channels", IEEE Journal on Selected Areas in Communications, vol.19, no.6, pp. 999-1008, 2001. 
[8] S. Chen, H. Shao and Q. Peng, "A new algorithm for symbol synchronization in DVB-T receiver", Electronic Engineer, vol. 33, no.9, pp. 33-40, 2007.

[9] Y. Li, "The Research and Design on Synchronization Algorithm for CMMB System Receiver", Beijing University of Posts and Telecommunications, 2009.
[10] H. Xie and Z. Tan, "Coarse symbol timing synchronization algorithm for CMMB transmission system", TV Technology, vol.35, no.2, pp. 22-25, 2011.

[11] P. Cheng, "Digital Signal Processing Course", Tsinghua University Press, 2001.

(C) Guoping et al.; Licensee Bentham Open.

This is an open access article licensed under the terms of the Creative Commons Attribution Non-Commercial License (http://creativecommons.org/licenses/by-nc/3.0/) which permits unrestricted, non-commercial use, distribution and reproduction in any medium, provided the work is properly cited. 